\title{
Análise da utilização do FMEA em projetos especiais de engenharia: Um estudo de
}

\section{caso em um carro de golfe autônomo}

Analysis of the use of FMEA in special engineering projects: A case study in an autonomous golf

cart

Análisis del uso de FMEA en proyectos especiales de ingeniería: Un caso de estudio en un carrito de golf autónomo

\author{
Bruno Junqueira Pereira \\ ORCID: https://orcid.org/0000-0001-9524-4793 \\ Universidade Federal de Itajubá, Brasil \\ E-mail: brunojunqueiracrv@gmail.com \\ Isabela Mansano de Siqueira \\ ORCID: https://orcid.org/0000-0002-2823-6926 \\ Universidade Federal de Itajubá, Brasil \\ E-mail: imansano09@gmail.com \\ Ana Carolina Oliveira Santos \\ ORCID: https://orcid.org/0000-0003-3313-1384 \\ Universidade Federal de Itajubá, Brasil \\ E-mail: anasantos@unifei.edu.br \\ Leonardo Geraldo Leite \\ ORCID: https://orcid.org/0000-0001-6312-2628 \\ Universidade Federal de Itajubá, Brasil \\ E-mail: leog.leite@hotmail.com \\ Hugo José Ribeiro Júnior \\ ORCID: https://orcid.org/0000-0002-1598-719X \\ Universidade Federal de Itajubá, Brasil \\ E-mail: hugojrr@unifei.edu.br
}

\begin{abstract}
Resumo
A Análise de Modos e Efeitos de Falha (FMEA) é uma ferramenta de gerenciamento de risco que oferece uma abordagem elaborada para avaliação, controle e atualização sobre o andamento de projetos, onde qualquer risco que afete qualidade, confiabilidade ou segurança deve ser avaliado e descrito no FMEA para prevenção de falhas. Os projetos de engenharia consistem em trabalhos desenvolvidos em faculdades por professores e alunos com a finalidade de desenvolver novas habilidades e competências. Além disso, estes projetos proporcionam aplicação prática de conhecimentos vistos na teoria, preparando os alunos para o mercado de trabalho, incentivando inovação e integração com questões sociais e ambientais. Este trabalho apresenta um estudo de caso sobre a utilização do FMEA no sistema de acoplamento e desacoplamento do volante na barra de direção de um projeto de engenharia que visa automatizar um carro de golfe. Os resultados encontrados consistem na identificação de modos de falhas e no cálculo nos seus respectivos RPN, bem como na elaboração de um plano de ação para reduzir ou eliminá-los. Este trabalho também constatou a importância de os graduandos conhecerem e utilizarem a ferramenta, dado que seu entendimento e aplicação também podem desenvolver competências e habilidades como análise crítica, pensamento sequenciado e ordenado, além de atuar na equipe com objetivo de eliminar desperdícios e otimizar projetos, processos, sistemas e serviços.
\end{abstract}

Palavras-chave: FMEA; Projetos especiais de engenharia; Modos de falhas; Qualidade.

\begin{abstract}
Failure Modes and Effects Analysis (FMEA) is a risk management tool that offers an elaborate approach to assess, control and update the progress of projects, where any risk that affects quality, reliability or safety must be assessed and described in FMEA for failure prevention. Engineering projects consist of work carried out in colleges by teachers and students to develop new skills and competences. In addition, these projects provide practical application of knowledge seen in theory, preparing students for the job market, encouraging innovation and integration with social and environmental issues. This work presents a case study on the use of FMEA in the steering wheel coupling and uncoupling system in the steering bar of an engineering project that aims to automate a golf cart. The results found consist of the identification of failure modes and the calculation of the respective RPN, as well as the elaboration of an action plan to reduce or eliminate it. This work also found the importance of graduating students to
\end{abstract}


know and use the tool, given that its understanding and application can also develop competencies and skills such as critical analysis, sequenced and ordered thinking, in addition to acting in the team with the objective of eliminating waste and optimizing projects, processes, systems and services.

Keywords: FMEA; Special engineering projects; Failure modes; Quality.

\section{Resumen}

El Análisis de Modos y Efectos de Falla (FMEA) es una herramienta de gestión de riesgos que ofrece un enfoque elaborado para evaluar, controlar y actualizar el progreso de los proyectos, donde cualquier riesgo que afecte la calidad, confiabilidad o seguridad debe ser evaluado y descrito en FMEA para la prevención de fallas. Los proyectos de ingeniería consisten en el trabajo realizado en las facultades por profesores y estudiantes con el fin de desarrollar nuevas habilidades y competencias. Además, estos proyectos proporcionan una aplicación práctica de los conocimientos vistos en teoría, preparando a los estudiantes para el mercado laboral, fomentando la innovación y la integración con los temas sociales y ambientales. Este trabajo presenta un caso de estudio sobre el uso de FMEA en el sistema de acoplamiento y desacoplamiento del volante en la barra de dirección de un proyecto de ingeniería que tiene como objetivo la automatización de un carrito de golf. Los resultados encontrados consisten en la identificación de modos de falla y el cálculo del respectivo RPN, así como la elaboración de un plan de acción para reducirlos o eliminarlos. Este trabajo también encontró la importancia de que los estudiantes graduados conozcan y utilicen la herramienta, dado que su comprensión y aplicación también pueden desarrollar habilidades como el análisis crítico, el pensamiento secuenciado y ordenado, además de trabajar en equipo con el objetivo de eliminar desperdicios y optimizar proyectos, procesos, sistemas y servicios.

Palabras clave: FMEA; Proyectos especiales de ingeniería; Modos de fallo; Calidad.

\section{Introdução}

A tecnologia evoluiu ao decorrer dos anos e com isso surgiram também novas metodologias de ensino para atender as demandas e exigências do mercado para formação de profissionais com capacidade de inovação e interação (Viana \& Silva, 2009). Assim, a educação brasileira em engenharia se depara com alguns desafios tais como: inovação, integração, preocupação com questões ambientais, sociais, culturais e contemporâneas, além de promover e possibilitar o desenvolvimento de diferentes habilidades e competências chamadas de transversais.

De acordo com Ibidem (2009) é importante a criação e estruturação de um ambiente de aprendizagem dentro da faculdade para que os alunos desenvolvam novas habilidades e competências tais como trabalho em equipe e capacidade de resolução de problemas, vivenciando, na prática, conteúdos que antes eram vistos somente na teoria. Isso possibilita um maior desenvolvimento da autonomia dos alunos, propiciando também experiência e simulação de cenários que podem ocorrer no ambiente de trabalho em que ele irá se inserir futuramente.

O método de Análise de Modos e Efeitos de Falha (FMEA) é uma ferramenta comumente utilizada em indústrias eletrônicas, automotivas, químicas, mecânicas, médicas, nucleares e aeroespaciais para identificar, priorizar e minimizar as falhas potenciais nos projetos de produto antes que esses sejam lançados no mercado (Yacoub \& Ammar, 2002).

Segundo Voltarelli (2018), o principal objetivo do FMEA consiste em reconhecer e priorizar falhas críticas, evidenciando os potenciais riscos e ajudando a construir um plano de ação que minimize as falhas detectadas. Por isso, pode-se verificar que a correta utilização do FMEA é fator importante para a melhoria dos indicadores de qualidade dos processos e projetos. Santos et al. (2020) afirmam que a ausência de falhas está diretamente à confiabilidade dos produtos, sendo esta um dos requisitos de valor para o cliente. Portanto, a prevenção de falhas utilizado nos projetos de produtos deve ser um item importante para a equipe de desenvolvimento.

Diante das vantagens da utilização do FMEA em novos projetos, esse trabalho pretende aplicar essa ferramenta em um projeto que está sendo desenvolvido por uma equipe composta por alunos e professores de alguns cursos de Engenharia da UNIFEI - Campus Itabira. O projeto consiste em transformar um carro de golfe elétrico em autônomo fazendo todas as adaptações necessárias para seu bom funcionamento.

Diante desse cenário, o presente trabalho tem por objetivo elaborar um FMEA no sistema de acoplamento e desacoplamento do volante na barra de direção do projeto de carro de golfe, identificar potenciais falhas que possam ocorrer 
após a instalação do novo sistema, propor planos de ação para diminuir a probabilidade de ocorrência das falhas ou aumentar a probabilidade de detecção dos modos de falhas que apresentarem maior índice de RPN, obter novos conhecimentos a respeito da ferramenta que é amplamente utilizada na indústria e analisar quais as reais contribuições da utilização dessa ferramenta para o desenvolvimento de projetos especiais de engenharia.

\section{Referencial teórico}

\subsection{Método de Análise de Modos e Efeitos de Falha (FMEA)}

Embora seja um método amplamente conhecido, a origem do FMEA ainda é imprecisa. Isso porque alguns autores acreditam que o FMEA foi criado em 1950 pela Grumman Aircraft Corporation, enquanto grande parte dos pesquisadores defende que militares norte-americanos utilizaram o FMEA pela primeira vez em 1949 e, em seguida, introduziram-no na indústria aeroespacial em meados da década de 60, durante a Missão Apollo. Segundo essa abordagem, a agência norteamericana NASA (National Aeronautics and Space Administration) teria criado um método com o intuito de identificar falhas potenciais em serviços, sistemas ou processos de forma sistemática, identificando assim as suas causas e efeitos. Dessa forma, tornou-se possível tomar decisões para eliminar ou, pelo menos, reduzir o risco das falhas que podem acontecer nos processos (Fernandes \& Rebelato, 2006).

O FMEA passou a ser empregado no mercado automobilístico a partir dos anos 80, depois que a Ford Motors Company foi a primeira empresa que passou a utilizar o método para fabricar seus automóveis (Clarke, 2005). Posteriormente começou a ser utilizado pelos fornecedores de autopeças. E hoje tem sido utilizado para analisar aspectos e impactos ambientais na indústria aeronáutica, posto que nem todos os riscos ambientais são facilmente identificados (Andrade, 2000). Vem sendo amplamente utilizado na indústria de manufatura em geral, para eliminar e reduzir falhas no desenvolvimento de produtos e no processo de fabricação (Galvani, 2013). Ainda segundo Galvani (2013), em síntese, a ferramenta FMEA pode ser utilizada para reconhecer riscos e priorizar ações para mitigar falhas em qualquer ocasião de produto, processo, sistema ou serviços.

Esta ferramenta utiliza três elementos como base: severidade, ocorrência e detecção. A partir deles é possível fazer análise da severidade da falha, ou seja, qual o impacto causado por ela, a probabilidade que ocorra e que seja detectada. Assim, por meio do FMEA é possível escolher os modos de falhas que apresentam maiores riscos ao funcionamento eficaz do processo, sistema ou serviço (Fernandes \& Rebelato, 2006). Segundo autores como Cassaneli et al. (2006) e Reid (2005), o FMEA permite quantificar os riscos a fim de garantir a qualidade de produtos e processos, sendo possível, preventivamente, remover ou mitigar falhas de maneira efetiva. Portanto, esta ferramenta é representada e entendida como uma sequência de fenômenos determinados como Causas, Falhas e Efeitos.

A classificação e medição da criticidade do risco de cada uma das falhas são feitos através do Número de Prioridade de Risco (Risk Priority Number - RPN). Para fazer a classificação dos riscos adota-se uma escala que varia de 1 a 10 pontos, na qual os avaliadores distribuem as notas. O RPN é o resultado da multiplicação dos três índices: Ocorrência $(\mathrm{O})$, Severidade (S) e Detecção (D). Os modos de falha que apresentarem maior valor de RPN devem ser priorizados e é necessário a criação de um plano de ação para correção e melhoria (Chang, 2009).

O índice de ocorrência segundo Tozzi (2004) está relacionado com a frequência com que as causas ou modos de falha ocorrem. Para se quantificar a frequência com que o problema pode ocorrer, utiliza-se a escala de avaliação apresentada no Quadro 1 - sendo que 1 representa ocorrência extremamente remota e 10 indica ocorrência certa. 
Quadro 1 - Avaliação da Ocorrência.

\begin{tabular}{|c|c|}
\hline ESCALA DE AVALIAÇÃO DA OCORR̂̂NCIA & GRAU \\
\hline Ocorrência certa & 10 \\
\hline Ocorrência muito elevada & 9 \\
\hline Ocorrência elevada & 8 \\
\hline Ocorrência frequente & 7 \\
\hline Ocorrência moderada & 6 \\
\hline Número ocasional de falhas & 5 \\
\hline Pequeno número de ocorrências & 4 \\
\hline Pequena chance de ocorrência & 3 \\
\hline Remoto, improvável & 2 \\
\hline Extremamente remoto & 1 \\
\hline
\end{tabular}

Fonte: Adaptado de Palady (1997).

A severidade, por sua vez, consiste na gravidade do efeito do modo de falha. Para que ela seja avaliada, utiliza-se as informações do Quadro 2 que consiste uma escala de 1 a 10, em que 1 representa que a falha não é percebida pelo cliente ou que é uma falha aceitável e sem maiores preocupações, ao passo que 10 indica os piores erros com suas piores consequências para o funcionamento do sistema (Palady, 1997). Ainda segundo Palady (1997), um valor alto nesse índice aponta que o cliente corre risco de segurança e que o custo alto da falha irá influenciar no bem-estar financeiro da organização.

Quadro 2 - Avaliação da severidade.

\begin{tabular}{|c|c|c|}
\hline EFEITO & SEVERIDADE DO EFEITO & GRAU \\
\hline $\begin{array}{l}\text { Perigoso, sem } \\
\text { aviso prévio }\end{array}$ & $\begin{array}{l}\text { Índice de severidade muito alto quando o modo de falha potencial afeta a } \\
\text { segurança na operação, sem aviso prévio. }\end{array}$ & 10 \\
\hline $\begin{array}{l}\text { Perigoso, com } \\
\text { aviso prévio }\end{array}$ & $\begin{array}{l}\text { Índice de severidade muito alto quando o modo de falha potencial afeta a } \\
\text { segurança na operação, com aviso prévio. }\end{array}$ & 9 \\
\hline Muito alto & $\begin{array}{l}\text { Severidade alta resultando em falha grave, porém não apresenta risco de } \\
\text { segurança }\end{array}$ & 8 \\
\hline Alto & $\begin{array}{l}\text { Efeito significativo, com nível de desempenho reduzido. Cliente muito } \\
\text { insatisfeito. }\end{array}$ & 7 \\
\hline Moderado & Efeito moderado, inconveniente para o cliente, acarretando na procura de serviço. & 6 \\
\hline Baixo & Efeito baixo, inconveniente para o cliente, porém não procura serviço. & 5 \\
\hline Muito baixo & $\begin{array}{l}\text { Efeito bastante insignificante, porém incomoda o cliente, causando a procura de } \\
\text { serviço. }\end{array}$ & 4 \\
\hline Menor & $\begin{array}{l}\text { Efeito bastante insignificante, porém incomoda o cliente, mas não ocasiona a } \\
\text { procura de serviço. }\end{array}$ & 3 \\
\hline Muito menor & Efeito muito insignificante na qual o cliente percebe, porém não procura serviço. & 2 \\
\hline Nenhum & Sem efeito identificado pelo cliente. & 1 \\
\hline
\end{tabular}

Fonte: Adaptado de Palady (1997). 
Por fim, a detecção está relacionada com a chance de detectar o modo de falha ou as causas que resultam nesse modo de falha. Encontram-se duas definições para a detecção (Palady, 1997): a chance de se detectar um problema antes que chegue ao cliente ou a chance de o cliente perceber um problema antes que provoque uma falha de consequência desastrosa. Para se realizar a classificação da detecção da falha, utiliza-se a escala apresentada no Quadro 3 que consiste no grau 1 para os problemas que são de deteç̧ão quase certa pelo controle atual até o grau 10 para os que não são possíveis de se identificar.

Quadro 3 - Avaliação da detecção.

\begin{tabular}{|c|c|}
\hline \multicolumn{1}{|c|}{ AVALIAÇÃO DE DETECÇÃ̃o } & GRAU \\
\hline $\begin{array}{c}\text { Não pode detectar ou não é verificado. Deteção quase impossível. O controle disponível não } \\
\text { detecta o modo de falha. }\end{array}$ & 10 \\
\hline Chance remota de detecção. A probabilidade do controle atual detectar a falha é muito pequena. & 9 \\
\hline Chance muito baixa de detecção pelo controle atual. & 8 \\
\hline Chance baixa de deteção pelo controle atual. & 7 \\
\hline Alguma probabilidade de detecção pelo controle atual. & 6 \\
\hline Probabilidade média de detecção pelo controle atual. & 5 \\
\hline Probabilidade moderada de detecção pelo controle atual. & 4 \\
\hline Probabilidade elevada de detecção pelo controle atual. & 3 \\
\hline Probabilidade de detecção muito elevada pelo controle atual. & 2 \\
\hline Detecção quase certa pelo controle atual. & 1 \\
\hline
\end{tabular}

Fonte: Adaptado de Palady (1997).

A causa e o efeito das falhas são relacionados entre si de forma organizada visto que os engenheiros responsáveis fazem a análise das causas raiz e efeitos finais das possíveis falhas. Deve-se sempre priorizar as falhas que possuírem maior valor de RPN, pois são as que apresentam maior risco ao bom funcionamento do sistema, processo e/ou produto (Yacoub \& Ammar, 2002). No entanto, os valores de RPN devem ser revisados, passando por novos cálculos após o plano de ação a fim de aferir se os riscos diminuíram e para examinar a real eficiência da ação corretiva para cada modo de falha (Liu et al., 2013).

Dessa maneira, verifica-se que o FMEA pode ser uma ferramenta útil para o aperfeiçoamento dos projetos especiais de engenharia. Isso porque além de auxiliar na priorização das melhorias no projeto, seu histórico pode promover informações sobre as ações implementadas e fomentar a implantação de uma cultura de gestão do conhecimento entre os discentes.

\subsection{Etapas do FMEA}

Segundo Fernandes e Rebelato (2006), o FMEA deve ser executado seguindo algumas etapas ordenadas, como:

a. Identificação de modos de falha potenciais e os já conhecidos;

b. Identificação dos efeitos dos modos de falha e sua severidade;

c. Identificação das possíveis causas dos modos de falha e a probabilidade que ocorram;

d. Identificação dos meios de detecção dos modos de falha a probabilidade de deteç̧ão;

e. $\quad$ Cálculo do RPN (Risk Priority Number);

f. Avaliação do potencial de risco dos modos de falha e definição de ações para reduzi-los ou eliminá-los. Isso é feito através de medidas que possibilitam diminuir a probabilidade de ocorrência da falha ou aumentar a probabilidade de detecção. 
A Figura 1 apresenta as etapas do FMEA de forma cíclica. Isso porque a partir da implementação das ações de melhoria, atualiza-se os modos de falhas existentes, os seus efeitos, causas e meios de deteç̧ão para então recalcula-se o novo RPN de modo a promover a melhoria contínua dos projetos e processos.

Figura 1 - Ciclo de etapas do FMEA.

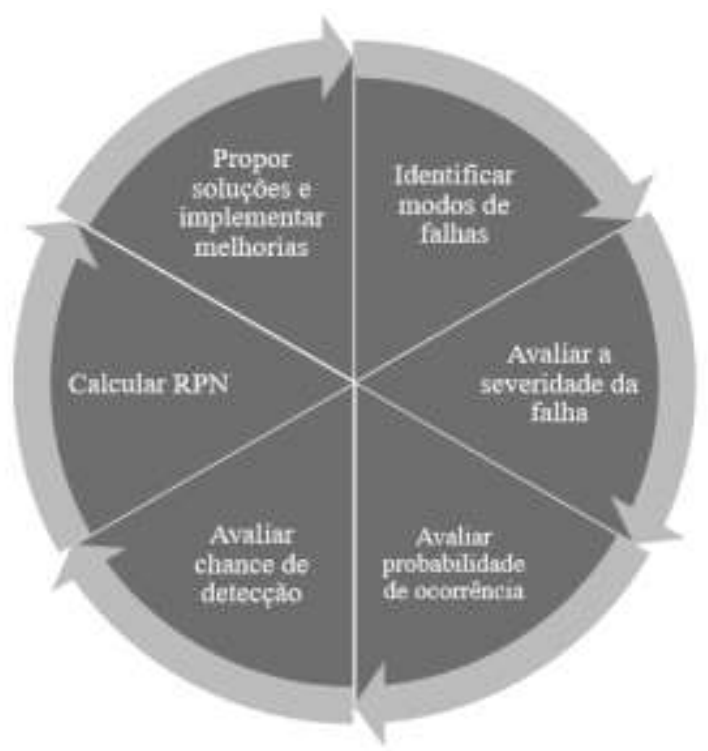

Fonte: Autores.

\subsection{Tipos de FMEA}

Dentre os tipos de FMEA existentes, vale ressaltar três: FMEA de processo, FMEA de sistema e FMEA de produto (Fernandes \& Rebelato, 2006).

Ainda segundo Fernandes e Rebelato (2006) o FMEA de produto é usado para identificação e avaliação de modos de falha no projeto de produto em situação anterior a disponibilização para a manufatura. Coloca em foco os modos de falha relacionados com a fidelidade aos objetivos preestabelecidos, expõe a carência de melhorias no projeto, ajuda a definir testes e formas de validar o produto, além de reconhecer as particularidades críticas e estimar possibilidades de alteração do projeto. O FMEA de produto considera e avalia todas as interações entre componentes, suas funções, os componentes presentes no sistema, as tolerâncias aceitáveis e todos os componentes utilizados (Bastos; 2006).

Segundo Bastos (2006), o FMEA de processo avalia os modos de falha em processos antes que sejam disponibilizados para produção. Expõe a carência de melhorias no processo, além de servir de apoio para analisar processos de montagem e manufatura e executar planos de controle. É feito através de fluxogramas do processo produtivo detalhado em cada etapa. Essa exposição ajuda a identificar potenciais variáveis para o processo de produção que necessitam de controle (Palady; 1997).

Segundo Fernandes e Rebelato (2006), o FMEA de sistema é usado para identificação e avaliação dos modos de falhas em sistemas nas primeiras fases de formulação e projeto. Coloca em foco os modos de falhas do sistema relacionado com suas funções. É utilizado na análise de subsistemas em fases iniciais de projeto e conceito, considera as interações entre os elementos que compõem o sistema e evidencia os potenciais modos de falha referente às funções do sistema (Rosa, 2005).

Entretanto, há autores que defendem a existência de apenas dois tipos. Conforme Renu (2016), o FMEA pode ser dividido em dois níveis tendo em vista sua utilização: FMEA de processo e FMEA de projeto. O FMEA de processo é usado para apurar a metodologia de produção e montagem para que seja possível fazer a identificação e análise dos modos de falha 
potenciais. Enquanto o FMEA de projeto enfoca a análise de subsistema e sistema do produto, procurando entender os possíveis modos de falha.

Por meio do embasamento teórico acerca dos tipos de FMEA chega-se à conclusão de que este presente trabalho se baseia no FMEA de projeto, visto que esta ferramenta irá apoiar estudos sobre a implantação de um novo sistema no carro de golfe antes da finalização da concepção do projeto. Sendo assim, com o auxílio desta metodologia pretende-se identificar e avaliar os modos de falha para reduzir ou eliminar potenciais falhas que possam ocorrer durante o desenvolvimento do projeto.

\section{Metodologia}

O presente trabalho tem por metodologia o Estudo de Caso, que é um método qualitativo que consiste em aprofundar o conhecimento sobre determinado tema, bem como identificar um problema, avaliar as evidências, desenvolver argumentos racionais, analisar e, por fim, propor soluções de melhoria. Nesse método, o pesquisador não tem muito controle sobre o fenômeno estudado e, tem por objetivo, propor melhorias para o problema encontrado (Miguel, 2007).

É classificado como Estudo de caso exploratório, por se tratar de uma abordagem de caráter qualitativo, entende-se a relevância de determinado fenômeno e sua interação com a população, visando prover uma base sólida para concepção de teorias e propostas de melhorias (Gil, 1996). Segundo Cervo e Silva (2006), o método exploratório define estratégias, procedimentos e métodos para buscar informações, elucidar o fenômeno e promover a criação de hipóteses e propostas de melhorias.

O estudo de caso é o método adequado para o presente trabalho, visto que o mesmo apresenta as etapas abordadas de forma clara. A característica mais relevante da metodologia no presente trabalho é que os dados foram coletados e analisados e posteriormente houve elaboração propostas de melhorias que foram passadas aos desenvolvedores do projeto especial de engenharia, porém se as ações serão implementadas é uma decisão dos alunos e professores envolvidos no Carro de golfe. Assim sendo, o problema foi detectado, analisado e proposto plano de ação, porém não executado, o que torna o estudo de caso o método mais adequado.

De acordo com Miguel (2007), as etapas de um Estudo de Caso consistem em: definir uma estrutura conceitualteórica; planejar o(s) caso(s); conduzir teste piloto; coletar os dados; analisar os dados e gerar relatório.

As duas primeiras etapas definidas para a elaboração de um estudo de caso, que consistem em definir a estruturação teórica e planejar o estudo de caso, estão apresentadas nos tópicos 1, 2 e 3 desse trabalho. Os tópicos a seguir apresentam a coleta de dados do estudo de caso único, análise dos dados e apresentação do relatório com as conclusões e sugestões de aplicações.

\section{Desenvolvimento}

\section{a. Objeto de estudo}

O objeto de estudo desse trabalho consiste no projeto de desenvolvimento de um carro de golfe elétrico para um sistema automatizado. O objetivo do projeto é fazer com que o carro de golfe seja capaz de se deslocar entre dois pontos utilizando somente os comandos dados pelo GPS. Este projeto está sendo desenvolvido por uma equipe composta por alunos e professores dos cursos de graduação de Engenharia Mecânica e da Computação da Universidade Federal de ItajubáCampus Itabira.

A ideia principal do projeto surgiu no segundo semestre do ano de 2016. Inicialmente os estudos começariam na parte computacional, onde seriam adaptados sensores, lasers e GPS para os comandos de navegação do carro. 
No entanto, foi verificada a necessidade de se ter um motor de passo para transmitir o movimento de esterçamento das rodas dianteiras através do acionamento polia-correia. Além disso, foi necessário desenvolver um suporte para esse motor de passo para que ele trabalhasse fixo e na angulação adequada. Duas polias foram acopladas nesse suporte: a polia menor está no suporte do motor de passo e a maior no eixo da cruzeta- o que transforma o comando em movimento do carro.

Outra necessidade foi identificada durante o desenvolvimento das atividades do projeto: um sistema de acoplamento do volante na barra de direção para facilitar e minimizar o risco de o volante mexer enquanto o carro estiver em movimento. Além disso, esse novo sistema poderia proporcionar a opção de mudança para controle manual ou automatizado do carro de golfe.

Essas duas alterações no projeto foram feiras no primeiro semestre de 2017 e agora a equipe trabalha no desenvolvimento da parte computacional, adaptando sensores, lasers e GPS para os comandos de navegação do carro.

O presente estudo tem por escopo a análise de modo e efeito de falhas no sistema mecânico do carro de golfe através da ferramenta FMEA. As Figuras 2A e 2B abaixo apresentam ao carro de golfe e a modificação feita no sistema. Nelas é possível verificar o sistema de acoplamento e desacoplamento da barra de direção do volante que foi escolhido dentro do sistema mecânico para uma análise confiável e detalhada.

Figura 2A: Carro de golfe.

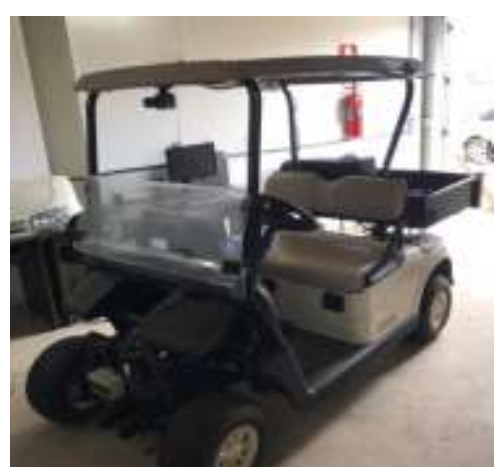

Fonte: Autores.
Figura 2B: Sistema de acoplamento e desacoplamento da barra de direção do volante.

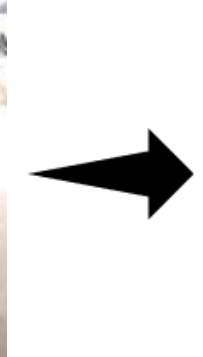

(1)

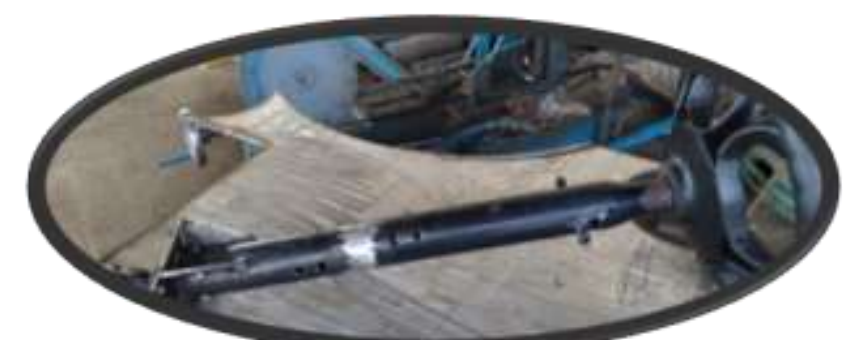

Fonte: Autores.

\section{b. Coleta de dados}

No início da coleta de dados foi feito um teste piloto com intuito de testar os procedimentos de aplicação dos conceitos do FMEA. A coleta de dados se iniciou com um brainstorming feito com um dos alunos desenvolvedores do projeto a fim de entender as mudanças que foram feitas, os sistemas que foram adaptados, qual a real necessidade de fazer cada alteração e adaptação para o funcionamento do carro. Durante essas reuniões as observações estavam sendo anotadas para posteriormente serem passadas para a tabela do FMEA.

A partir do momento que foi possível compreender o sistema e todas as adaptações que tinham sido feitas, iniciou-se o preenchimento da tabela. Inicialmente foi utilizada uma tabela para elaboração do FMEA mais simples com poucas funções somente para preenchimento. Visto a necessidade de identificação das ações a serem priorizadas a tabela foi formatada para o RPN ser calculado através de fórmulas e as células serem coloridas automaticamente de acordo com os conceitos do FMEA.

Houve a necessidade de inúmeras reuniões de alinhamento dos autores deste trabalho com os outros desenvolvedores para que fosse possível analisar todas as possíveis falhas e modos de falha que podem ocorrer, desde as que apresentavam chances mais remotas até as que possuem falhas críticas e evidentes. Após vários encontros e reuniões, foram feitos ajustes 
na tabela para se tornar o mais fidedigno possível acerca dos possíveis modos de falhas, causas, efeitos e, no final, foi possível traçar um plano de ação para cada um dos modos que apresentaram maior índice de RPN e devem ser analisados para prevenir ou eliminar a ocorrência de falhas.

\section{c. Elaboração do FMEA}

Como o primeiro objetivo desse trabalho consiste em utilizar o FMEA no projeto de engenharia "Carro de Golfe", a elaboração do FMEA foi realizada pelos autores desse trabalho, juntamente a equipe do projeto que auxiliou na montagem da tabela do FMEA através de várias reuniões.

Nessas reuniões foram identificados os potenciais modos de falha e os já conhecidos, suas possíveis causas e as probabilidades que ocorram, bem como os efeitos e a severidade dos mesmos. Foram identificados, também, meios de detecção dos modos de falha a probabilidade de detecção. Foi feita a avaliação do potencial de risco dos modos de falha e definição de ações para reduzi-los ou eliminá-los. Isso é feito através de medidas que possibilitam diminuir a probabilidade de ocorrência da falha ou aumentar a probabilidade de detecção. Dessa forma, a planilha do FMEA foi preenchida, os principais modos de falha identificados na função de acoplamento e desacoplamento da haste do volante à barra de direção foram: mola da haste romper, mola da haste perder a elasticidade, parafuso do engate romper, arruela lisa limitadora da mola estar mal fixada, correia dentada romper e cruzeta emperrar, cruzeta romper; polias estar desalinhadas, chaveta romper, desgaste e/ou quebra do soquete, má fixação do soquete e a interrupção do funcionamento do motor de passo.

O Quadro 4 apresenta o FMEA parcial com os componentes e modos de falhas suscetíveis a erros que necessitaram de elaboração de plano de ação para o sistema de acoplamento da barra de direção no volante. Nele são apresentados alguns dos componentes do equipamento (mola da haste, parafuso de acionamento e arruela lisa) suas falhas potenciais, bem como os seus respectivos índices de severidade, ocorrência, detecção e RPN. Os itens destacados em vermelho são os que apresentaram maiores valores para severidade e os em amarelos são os que obtiveram os maiores valores de RPN. Para todos os itens foram propostas ações para mitigar a causa raiz da possível falha.

O mesmo FMEA se replica para o sistema de desacoplamento, pois os componentes e modos de falhas encontrados são os mesmos. O documento completo com a análise de todos os modos de falhas encontra-se disponível no link: https://figshare.com/articles/dataset/_/14256536.

\section{d. Análise dos dados e geração de relatórios.}

O FMEA identificou doze (12) possíveis modos de falhas, categorizados pelas cores vermelho, amarelo e laranja. A cor vermelha representa um risco de segurança e, portanto, independente da pontuação, deve ser gerado um plano de ação para mitigá-los. A cor amarela indica riscos considerados como médios, com pontuação média, mas que devem ser tratados por meio de plano de ação. A cor laranja (que consta do FMEA completo disponibilizado online) demonstra que aquele modo de falha é considerado de alto risco, ou seja, sua pontuação é alta e, por isso, deve-se elaborar um plano de ação para prevenir que a falha aconteça.

As partes mecânicas que mostraram ser suscetíveis a falhas após a etapa de definição e projeto, bem como suas funções, foram:

- Mola da haste - Permitir a transferência da direção entre comando manual e automatizado, através do encaixe interno por meio do parafuso de acionamento;

- $\quad$ Parafuso de acionamento - Permitir o acoplamento e desacoplamento do sistema de direção através de engate;

- $\quad$ Arruela lisa - Limitar o espaço percorrido pela mola para que ela não adentre no cachimbo; 
- Correia dentada - Conectar a polia menor do motor de passo com a polia maior que conecta à haste do volante para rotacionar o volante;

- Cruzeta - Transmitir rotação de eixo em ângulos distintos;

- $\quad$ Polias - Transmitir força e movimento;

- Chavetas - Travar o elemento rotativo no eixo;

- $\quad$ Soquete cromado 19mm - Conectar/desconectar o parafuso da barra de direção;

- Motor de passo - Transmitir o movimento de esterçamento das rodas dianteiras, através do acionamento do conjunto de polias-correia.

Quadro 4 - Tabela do FMEA para modos de falha críticos.

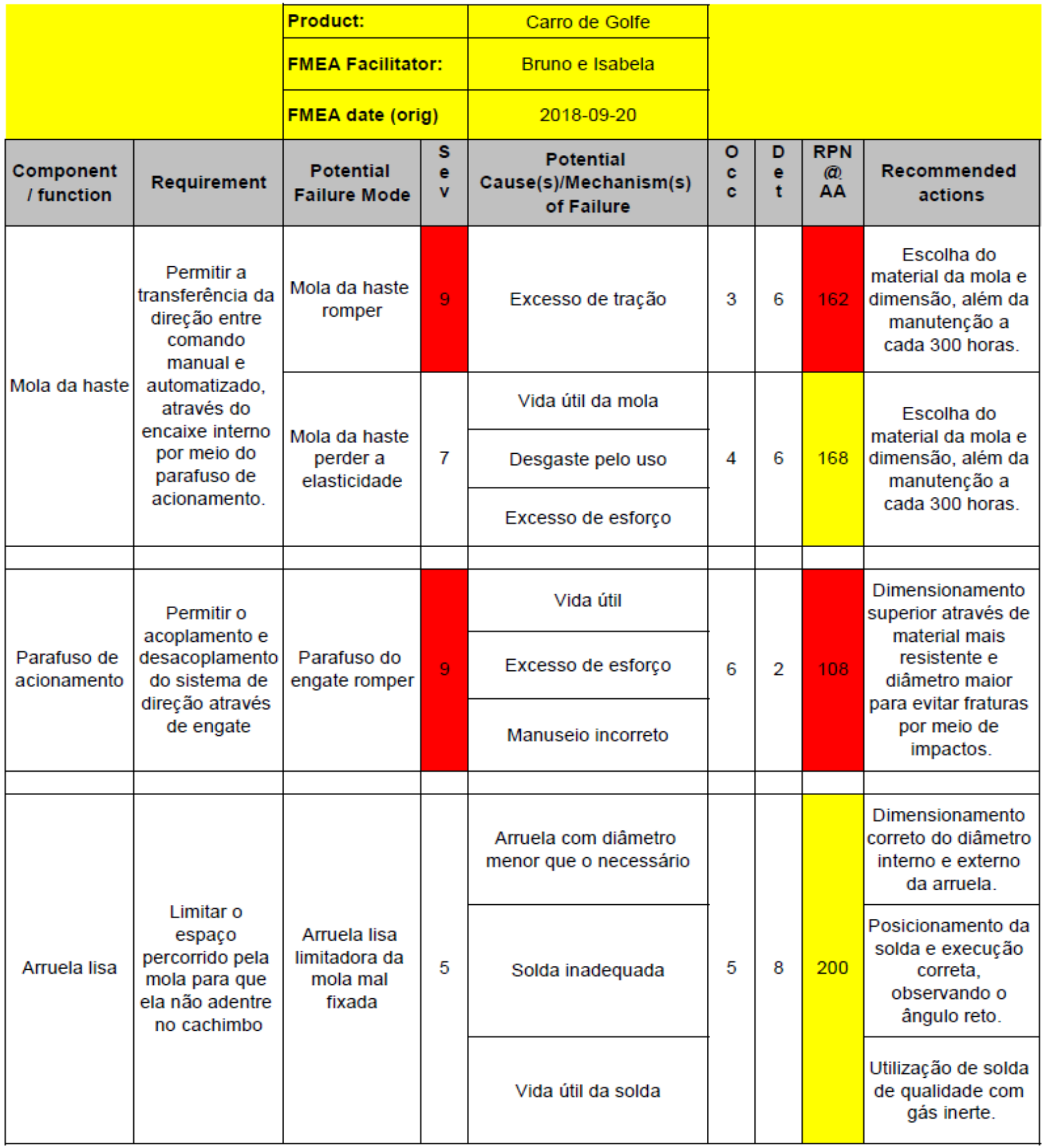


As Figuras 3A e 3B a seguir ilustram os componentes e a respectiva localização no sistema de acoplamento e desacoplamento do volante da barra de direção, de acordo com a numeração utilizada no FMEA.

Figura 3A - Componentes do sistema.

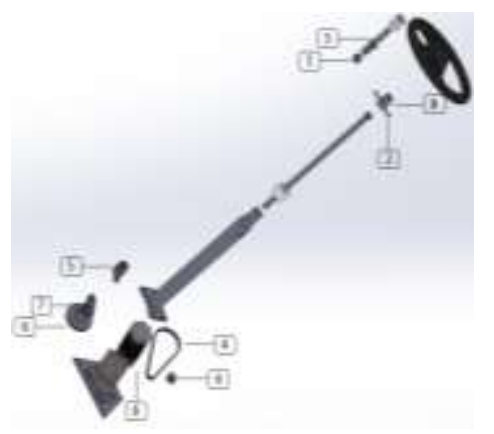

Fonte: Adaptado do solidworks fornecido pelos desenvolvedores.

Figura 3B - Montagem do sistema.

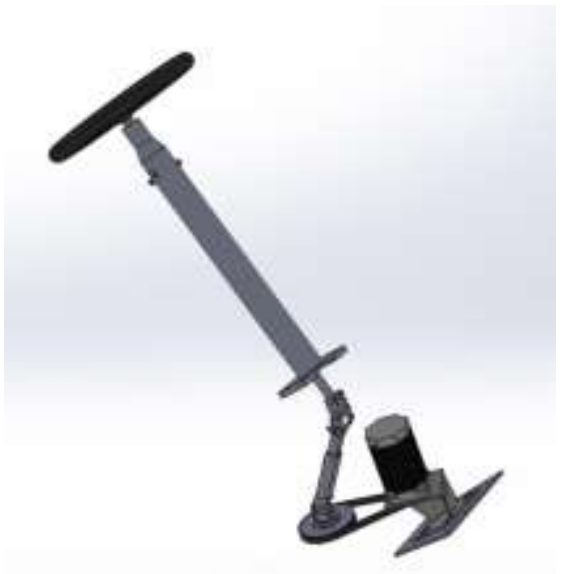

Fonte: Adaptado do solidworks fornecido pelos desenvolvedores.

O Quadro 5 foi apresenta o plano de ação que foi elaborado após identificar os componentes e suas possíveis causas, dessa maneira foi possível consolidar todas as ações necessárias para a prevenção dos modos de falhas identificados. 
Quadro 5 - Plano de ação.

\begin{tabular}{|c|c|c|c|c|c|}
\hline COMPONENTE & $\begin{array}{l}\text { POTENCIAL } \\
\text { CAUSA }\end{array}$ & AÇÃO RECOMENDADA & RESPONSÁ VEL & PRAZO & STATUS \\
\hline \multirow{4}{*}{ Mola da haste } & Excesso de tração & \multirow{4}{*}{$\begin{array}{l}\text { Escolha do material da mola e } \\
\text { dimensão, além da manutenção a } \\
\text { cada } 300 \text { horas. }\end{array}$} & \multirow{4}{*}{ "A definir" } & \multirow{4}{*}{$\begin{array}{c}\text { "A } \\
\text { definir" }\end{array}$} & \multirow{4}{*}{ Follow up } \\
\hline & Vida útil da mola & & & & \\
\hline & Desgaste pelo uso & & & & \\
\hline & Excesso de esforço & & & & \\
\hline \multirow{3}{*}{$\begin{array}{l}\text { Parafuso de } \\
\text { acionamento }\end{array}$} & Vida útil & \multirow{3}{*}{$\begin{array}{l}\text { Dimensionamento superior } \\
\text { através de material mais } \\
\text { resistente e diâmetro maior para } \\
\text { evitar fraturas por meio de } \\
\text { impactos. }\end{array}$} & \multirow{3}{*}{ "A definir" } & \multirow{3}{*}{$\begin{array}{c}\text { "A } \\
\text { definir" }\end{array}$} & \multirow{3}{*}{ Follow up } \\
\hline & Excesso de esforço & & & & \\
\hline & Manuseio incorreto & & & & \\
\hline \multirow{3}{*}{ Arruela lisa } & $\begin{array}{l}\text { Arruela com diâmetro } \\
\text { menor que o } \\
\text { necessário }\end{array}$ & $\begin{array}{l}\text { Dimensionamento correto do } \\
\text { diâmetro interno e externo da } \\
\text { arruela. }\end{array}$ & "A definir" & $\begin{array}{c}\text { "A } \\
\text { definir" }\end{array}$ & Follow up \\
\hline & Solda inadequada & $\begin{array}{c}\text { Posicionamento da solda e } \\
\text { execução correta, observando o } \\
\text { ângulo reto. } \\
\end{array}$ & "A definir" & $\begin{array}{c}\text { "A } \\
\text { definir" }\end{array}$ & Follow up \\
\hline & Vida útil da solda & $\begin{array}{l}\text { Utilização de solda de qualidade } \\
\text { com gás inerte. }\end{array}$ & "A definir" & $\begin{array}{c}\text { "A } \\
\text { definir" }\end{array}$ & Follow up \\
\hline \multirow[t]{2}{*}{ Correia dentada } & Vida útil da correia & $\begin{array}{c}\text { Garantir o correto alinhamento } \\
\text { da correia e manutenção } \\
\text { preventiva. } \\
\end{array}$ & "A definir" & $\begin{array}{c}\text { "A } \\
\text { definir" }\end{array}$ & Follow up \\
\hline & Excesso de esforço & $\begin{array}{c}\text { Isolar o sistema para evitar } \\
\text { contato direto com água e poeira. }\end{array}$ & "A definir" & $\begin{array}{c}\text { "A } \\
\text { definir" }\end{array}$ & Follow up \\
\hline \multirow{7}{*}{ Cruzeta } & $\begin{array}{l}\text { Falta de lubrificação } \\
\text { ou lubrificação } \\
\text { incorreta }\end{array}$ & $\begin{array}{c}\text { Manutenção preventiva por meio } \\
\text { de plano de manutenção } \\
\text { elaborado em horas de } \\
\text { funcionamento. } \\
\end{array}$ & "A definir" & $\begin{array}{c}\text { "A } \\
\text { definir" }\end{array}$ & Follow up \\
\hline & $\begin{array}{l}\text { Contaminação por } \\
\text { água } \\
\end{array}$ & Isolar de contato externo. & "A definir" & $\begin{array}{c}\text { "A } \\
\text { definir" }\end{array}$ & Follow up \\
\hline & $\begin{array}{l}\text { Cargas de torque } \\
\text { excessivas }\end{array}$ & \multirow{2}{*}{$\begin{array}{l}\text { Utilização por meio do condutor } \\
\text { e materiais nobres dependendo } \\
\text { da utilização do carro. }\end{array}$} & "A definir" & $\begin{array}{c}\text { "A } \\
\text { definir" }\end{array}$ & Follow up \\
\hline & $\begin{array}{l}\text { Cargas de choque } \\
\text { excessivas }\end{array}$ & & "A definir" & $\begin{array}{c}\text { "A } \\
\text { definir" } \\
\end{array}$ & Follow up \\
\hline & $\begin{array}{l}\text { Aplicação inadequada } \\
\text { devido a maior } \\
\text { angulação que o } \\
\text { especificado } \\
\end{array}$ & $\begin{array}{c}\text { Dimensionamento da cruzeta de } \\
\text { acordo com o projeto a ser } \\
\text { utilizada. }\end{array}$ & "A definir" & $\begin{array}{c}\text { "A } \\
\text { definir" }\end{array}$ & Follow up \\
\hline & $\begin{array}{c}\text { Ângulos de operação } \\
\text { excessivos }\end{array}$ & Não operar a mais de $40^{\circ}$. & "A definir" & $\begin{array}{c}\text { "A } \\
\text { definir" }\end{array}$ & Follow up \\
\hline & $\begin{array}{c}\text { Procedimentos de } \\
\text { montagem } \\
\text { inadequado } \\
\end{array}$ & Especificação do projeto. & "A definir" & $\begin{array}{c}\text { "A } \\
\text { definir" }\end{array}$ & Follow up \\
\hline Polias & $\begin{array}{l}\text { Procedimento de } \\
\text { montagem } \\
\text { inadequado }\end{array}$ & $\begin{array}{l}\text { Criação de gabarito poka yoke } \\
\text { específico para montagem das } \\
\text { polias para correta angulação e } \\
\text { alinhamento. }\end{array}$ & "A definir" & $\begin{array}{c}\text { "A } \\
\text { definir" }\end{array}$ & Follow up \\
\hline \multirow[t]{2}{*}{ Chaveta } & $\begin{array}{c}\text { Material inadequado } \\
\text { para a interação com } \\
\text { o material da outra } \\
\text { peça } \\
\end{array}$ & \multirow{2}{*}{$\begin{array}{c}\text { Escolha de material adequado } \\
\text { pois sua função é romper antes } \\
\text { que danifique outros } \\
\text { componentes. }\end{array}$} & "A definir" & $\begin{array}{c}\text { "A } \\
\text { definir" }\end{array}$ & Follow up \\
\hline & Força excessiva & & "A definir" & $\begin{array}{c}\text { "A } \\
\text { definir" }\end{array}$ & Follow up \\
\hline
\end{tabular}




\begin{tabular}{|c|c|c|c|c|c|}
\hline \multirow{2}{*}{$\begin{array}{l}\text { Soquete } \\
\text { Cromado 19mm }\end{array}$} & $\begin{array}{l}\text { Torque excessivo/uso } \\
\text { constante }\end{array}$ & $\begin{array}{l}\text { Dimensionamento superior e } \\
\text { manutenção através de plano de } \\
\text { acordo com horas de utilização. }\end{array}$ & "A definir" & $\begin{array}{c}\text { "A } \\
\text { definir" }\end{array}$ & Follow up \\
\hline & Solda incorreta & $\begin{array}{l}\text { Utilização de solda de qualidade } \\
\text { com gás inerte. }\end{array}$ & "A definir" & $\begin{array}{c}\text { "A } \\
\text { definir" }\end{array}$ & Follow up \\
\hline Motor de Passo & $\begin{array}{l}\text { Contato direto com } \\
\text { água }\end{array}$ & $\begin{array}{l}\text { Manter motor isolado com } \\
\text { blindagem correta através de } \\
\text { proteção IP e isolar sistema de } \\
\text { contato direto com água. }\end{array}$ & "A definir" & $\begin{array}{c}\text { "A } \\
\text { definir" }\end{array}$ & Follow up \\
\hline
\end{tabular}

Fonte: Autores.

\section{e. Contribuições para o desenvolvimento de projetos de engenharia}

A partir dos dados coletados, análises feitas e plano de ação gerado, pôde-se ressaltar a importância da ferramenta FMEA no desenvolvimento de projetos especiais de engenharia liderados por alunos, professores e técnicos de Universidades e Faculdades. No projeto em questão foi essencial para identificar as possíveis falhas citadas nos tópicos anteriores, porém, em outros projetos, pode-se pensar na escolha dos materiais mais adequados e suas interações, escolha dos pneus para diferentes climas, se a bateria é suficiente, entre outros.

A aplicação do FMEA nos projetos de competição certamente traria resultados tangíveis nas competições, uma vez que seria possível rastrear os defeitos já ocorridos no passado, identificar novos potenciais e trabalhar preventivamente para que não ocorram no dia da prova. É uma ferramenta de melhoria contínua que busca sempre a potencialização da qualidade e, ao mesmo tempo, possibilita categorizar, de forma sistemática, as informações sobre falhas e ter melhor conhecimento dos problemas oriundos do projeto ou processo.

Além disso, em reunião com os desenvolvedores do projeto do carro foi possível notar que alguns deles não tinham conhecimento sobre a existência desta ferramenta e que, quando entenderam o funcionamento, suas vantagens e como ela poderia auxiliar no desenvolvimento de projetos, eles viram e citaram a importância e relevância do FMEA não só para o projeto desenvolvido por eles, mas para outros trabalhos que são feitos dentro da faculdade. Foi comentado também sobre o quão seria interessante que os alunos de outros cursos tivessem conhecimento sobre a existência dessa ferramenta para análise, identificação, prevenção e priorização de falhas que podem ocorrer em projetos especiais de engenharia.

Os desenvolvedores do projeto especial de engenharia citaram ainda que isso poderia influenciar, como dito anteriormente pelos autores, na eficácia dos projetos e nos resultados das competições, pois facilitaria a identificação e prevenção de falhas no sistema. É importante ressaltar que, segundo eles, é de grande valor o estudo realizado e que pretendem utilizar em projetos futuros.

\section{Conclusão}

O desenvolvimento do presente estudo possibilitou a aplicação da ferramenta FMEA no projeto especial de engenharia, carro de golfe. Após a condução do teste piloto e as melhorias realizadas na tabela de coleta de dados foi possível identificar potenciais falhas, priorizar os riscos e elaborar um plano de ação para eliminá-los. Durante a realização do teste piloto foi possível identificar melhorias que poderiam auxiliar na montagem da tabela, tornando-a mais interativa e dinâmica.

As coletas de dados foram realizadas por meio de reuniões com os desenvolvedores do novo sistema. A maior dificuldade encontrada na realização do presente trabalho consistiu no fato de que o FMEA deve ser elaborado por meio de uma equipe multidisciplinar e deve ser conduzido por um engenheiro de validação devidamente treinado e certificado. Dessa forma, foi um desafio para os autores aplicar a ferramenta com uma equipe reduzida e pouco interdisciplinar, já que as 
reuniões eram compostas apenas por graduandos em Engenharia Mecânica e de Produção, além de um mestrando em Engenharia mecânica, pessoas que possuíam conhecimento sobre o desenvolvimento e alterações do projeto Carro de Golfe.

Vale ressaltar que apesar de algumas dificuldades encontradas, foi possível obter novos conhecimentos sobre a ferramenta FMEA que é amplamente utilizada na indústria de diversos setores, além da confirmação que ela é relevante e aplicável nos projetos especiais de engenharia a fim de eliminar ou reduzir falhas que possam ocorrer durante o desenvolvimento dos projetos ou em competições. Faz parte da melhoria contínua e categorização de prioridades a serem atacadas. Além disso, é de suma importância que graduandos de todos os cursos conheçam e utilizem a ferramenta, dado que é possível rastrear defeitos já ocorridos no projeto, identificar novos e trabalhar preventivamente. O contato e entendimento da ferramenta FMEA desenvolve competências e habilidades como análise crítica, pensamento sequenciado e ordenado, visando eliminar desperdícios e otimizar projetos, processos, sistemas e serviços.

Por fim, pode-se concluir que o FMEA permite a identificação de potenciais falhas antes que elas aconteçam, convergindo com os princípios da metodologia Lean Manufacturing que, embora não seja o foco do artigo. Dessa maneira, sugere-se como trabalhos futuros análise da utilização do FMEA nos projetos de engenharia, tais como redução de não conformidades e melhoria da qualidade e a confiabilidade do produto ou serviço.

\section{Referências}

Andrade, M. R. S., \& Turrioni, J. B. (2000). Uma metodologia de análise dos aspectos e impactos ambientais através da utilização do FMEA. XX Encontro Nacional de Engenharia de Produção - ENEGEP, São Paulo - SP.

Bastos, A. (2006). FMEA como ferramenta de prevenção da qualidade em produtos e processos-uma avaliação da aplicação em um processo produtivo de usinagem de engrenagem. XXVI Encontro Nacional de Engenharia de Produção - ENEGEP, Fortaleza - CE.

Cassanelli, G., Mura, G., Fantini, F., Vanzi, M., \& Plano, B. (2006). Failure analysis-assisted FMEA. Microelectronics Reliability, 46(9-11), 1795-1799.

Chang, K. H. (2009). Evaluate the orderings of risk for failure problems using a more general RPN methodology. Microelectronics Reliability, 49 (12), 15861596.

Clarke, C. (2005). Automotive production systems and standardisation: from Ford to the case of Mercedes-Benz. Springer Science \& Business Media ( $1^{\mathrm{a}}$.Ed.). German: Physica-Verlag Heidelberg.

Fernandes, J. M. R., \& Rebelato, M. G. (2006). Proposta de um método para integração entre QFD e FMEA. Gestão \& Produção, 13(2), $245-259$.

Galvani, L. R., \& Carpinetti, L. C. R. (2013). Análise comparativa da aplicação do programa Seis Sigma em processos de manufatura e serviços. Production, 23(4), 695-704.

Gil, A. C. (1996). Como elaborar projetos de pesquisa (3a ed.), Atlas.

Liu, H. C., Liu, L., \& Liu, N. (2013). Risk evaluation approaches in failure mode and effects analysis: A literature review. Expert systems with applications, 40(2), 828-838.

Miguel, P. A. C. (2007). Estudo de caso na engenharia de produção: estruturação e recomendações para sua condução. Production, 17(1), 216-229.

Palady, P. (2004). FMEA: Análise dos Modos de Falha e Efeitos: prevendo e prevenindo problemas antes que ocorram (3a ed.) IMAM.

Reid, R. D. (2005). FMEA — something old, something new. Quality Progress, 38(5), 90-93.

Renu, R., Visotsky, D., Knackstedt, S., Mocko, G., Summers, J. D., \& Schulte, J. (2016). A knowledge based FMEA to support identification and man agement of vehicle flexible component issues. Procedia Cirp, 44, 157-162.

Rosa, C. B. (2005). Practical application of FMEA and QFD to improve product quality (No. 2005-01-3972). SAE Technical Paper.

Santos, A. C. O., da Silva, C. E. S., Braga, R. A. D. S., Corrêa, J. É., \& de Almeida, F. A. (2020). Customer value in lean product development: Conceptual model for incremental innovations. Systems Engineering, 23(3), 281-293.

Tozzi, A. R. (2004). Desenvolvimento de um programa de verificação de um processo de lançamento de cabos com o auxílio da FMEA. Dissertação de Mestrado. Universidade Federal do Rio Grande do Sul - Escola de Engenharia, Porto Alegre - RS, Brasil.

Viana, D. M., \& Silva, M. D. F. S. (2011). Projeto de um veículo elétrico para apoio à coleta seletiva: uma experiência que une ensino, pesquisa e extensão. In: Catalão, V. M. L., Layrargues, P. P., \& Zaneti, I.C.B.B. (Org.). Universidade para o século XXI: educação e gestão ambiental na Universidade de Brasília. Brasília: Cidade Gráfica e Editora. 
Research, Society and Development, v. 10, n. 4, e5701048448, 2020

(CC BY 4.0) | ISSN 2525-3409 | DOI: http://dx.doi.org/10.33448/rsd-v10i4.8448

Voltarelli, M. A., Paixão, C. S., Zerbato, C., Silva, R. P. D., \& Gazzola, J. (2018). Failure mode and effect analysis (FMEA) in mechanized harvest of sugarcane billets. Engenharia Agrícola, 38(1), 88-96.

Yacoub, S. M., \& Ammar, H. H. (2002). A methodology for architecture-level reliability risk analysis. IEEE Transactions on Software engineering, 28(6), 529-547. 